\title{
Energetic Quantum Limit in Large-Scale Interferometers
}

\author{
Vladimir B. Braginsky, Mikhail L. Gorodetsky, \\ Farid Ya. Khalili, ${ }^{*}$ and Kip S. Thorne ${ }^{\dagger}$ \\ *Physics Faculty, Moscow University, Moscow Russia \\ $\dagger$ Theoretical Astrophysics, California Institute of Technology, Pasadena, CA 91125
}

\begin{abstract}
Abstraet. For each optical topology of an interferometric gravitational wave detector, quantum mechanics dictates a minimum optical power (the "energetic quantum limit") to achieve a given sensitivity. For standard topologies, when one seeks to beat the standard quantum limit by a substantial factor, the energetic quantum limit becomes impossibly large. Intracavity readout schemes may do so with manageable optical powers.
\end{abstract}

\section{THE ENERGETIC QUANTUM LIMIT}

It is well known that quantum mechanics limits the sensitivity of traditional position measurements by the Standard Quantum Limit (SQL). Several methods of overcoming the SQL have been proposed. There is almost no doubt that in the next several years large-scale gravitational wave antennae will reach the level of the SQL and possibly will beat it by factor $2 \div 3$. Are there any other quantum limits beyond the SQL?

One possible answer is: the next serious limitation is the Energetic Quantum Limit. A gravitational wave in an interferometric antenna changes the phase of the optical field. In order to detect this phase shift, the uncertainty of the phase $\Delta \phi$ must be sufficiently small. In particular, due to the uncertainty relation

$$
\Delta \mathcal{E} \Delta \phi \geq \frac{\hbar \omega_{0}}{2}
$$

(where $\omega_{0}$ is the optical frequency), a large uncertainty of the optical energy $\mathcal{E}$ is required.

This is not a peculiar property of interferometric meters only, but a consequence of a more general principle: In order to detect an external action on a quantum object, the uncertainty of the interaction Hamiltonian $\hat{\mathcal{H}}_{I}$ must be sufficiently large [1-3]:

$$
\left\langle\left(\int_{-\infty}^{\infty} \Delta \mathcal{H}_{I}(t) d t\right)^{2}\right\rangle \geq \frac{\hbar^{2}}{4} .
$$

The uncertainty of $\mathcal{H}_{I}$ is related to the signal-to-noise ratio by

CP523, Gravitational Waves: Third Edoardo Amaldi Conference, edited by S. Meshkov ○ 2000 American Institute of Physics 1-56396-944-0/00/\$17.00 


$$
\frac{S}{N}=\frac{4}{\hbar^{2}}\left\langle\left(\int_{-\infty}^{\infty} \Delta \mathcal{H}_{I}(t) d t\right)^{2}\right\rangle
$$

For laser interferometer gravitational-wave antennas this is equivalent to

$$
\frac{S}{N}=\frac{4}{\hbar^{2} L^{2}} \int_{-\infty}^{\infty} x_{\text {signal }}(t) x_{\text {signal }}\left(t^{\prime}\right) B_{\mathcal{E}}\left(t, t^{\prime}\right) d t d t^{\prime},
$$

where $L$ is the length of the arms of the antenna,

$$
x_{\text {signal }}(t)=\frac{L h(t)}{2}
$$

is the effective change of $L$ caused by a gravitational wave, $h(t)$ is the variation of the wave's metric, and $B_{\mathcal{E}}\left(t, t^{\prime}\right)$ is the correlation function of the optical energy in the antenna, or the correlation function of the difference of energies in the two arms of the antenna if two-arm-topology is used.

The origin of the limitation (2) is the Heisenberg uncertainty relation. To detect a small displacement of the mirrors, it is necessary to apply to them a sufficiently strong random kick. The only source of this kick is the uncertainty of the optical energy in the antenna or of the difference of energies in the two arms.

We shall limit ourselves here to the stationary regime, for which the quantum state of the electromagnetic field in the interferometer does not depend explicitly on time. In this case formula (4) can be rewritten in spectral form as

$$
\frac{S}{N}=\frac{4}{\hbar^{2} L^{2}} \int_{-\infty}^{\infty}\left|X_{\text {signal }}(\omega)\right|^{2} S_{\mathcal{E}}(\omega) \frac{d \omega}{2 \pi},
$$

where $X_{\text {signal }}(\omega)$ is the Fourier transform of $x_{\text {signal }}(t)$, and $S_{\mathcal{E}}(\omega)$ is the spectral density of the fluctuations of the optical energy. It is important to note here that formula (4) is the ultimate limit on the sensitivity for any measurement technique, and formula (6) describes the ultimate sensitivity for all stationary procedures.

\section{COMPARISON WITH THE SQL}

In all estimates below we will use the value of the Standard Quantum Limit (SQL) as a convenient measure of sensitivity. The SQL, as it was defined more than thirty years ago [4], is the sensitivity of an ordinary position meter, i.e. a position meter which does not use any non-stationary or correlation methods to increase the sensitivity. The forms of the SQL as usually given in the literature, are not convenient since they are based on some assumed shape of the force's time dependence (most commonly a singlecycle sinusoid or a long, monochromatic wave train). Here we prefer a more general form of the SQL expressed in terms of the spectral density $S(\omega)$ for the net noise of a measurement device. This (double-sided) spectral density is defined in such a way that for optimal signal processing the signal to noise ratio is equal to

$$
\frac{S}{N}=\int_{-\infty}^{\infty} \frac{\left|X_{\text {signal }}(\omega)\right|^{2}}{S(\omega)} \frac{d \omega}{2 \pi}
$$

In the case of an ordinary position meter the spectral density of the net noise is equal to 


$$
S_{S Q L}(\omega)=S_{x}+\frac{S_{F}}{M^{2} \omega^{4}}
$$

where $M$ is the test mass, $S_{x}(\omega)$ is the spectral density of the noise that the meter superimposes on the output position signal, $S_{F}(\omega)$ is the spectral density of the fluctuating back-action force that the meter exerts on the test mass, and these noises satisfy the uncertainty relation [5],

$$
S_{x} S_{F} \geq \hbar^{2} / 4
$$

We assume that the position meter is a perfect one, corresponding to equality in this uncertainty relation, and the spectrum of the signal is concentrated near the frequency

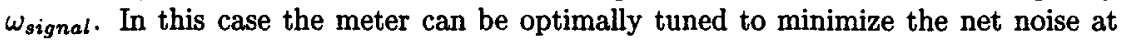
this frequency:

$$
S_{S Q L}\left(\omega_{\text {signal }}\right)=\frac{\hbar}{M \omega_{s i g n a l}^{2}}
$$

As follows from formulas (6), (7) and (10), in order to beat this SQL for the spectral density of $x_{\text {signal }}$ by a factor $\xi^{2}<1$, the spectral density of the fluctuations of the optical energy must obey the condition

$$
S_{\mathcal{E}}\left(\omega_{\text {signal }}\right) \geq \frac{\hbar^{2}}{S_{S Q L}\left(\omega_{\text {signal }}\right) \xi^{2}}=\frac{\hbar M \omega_{s i g n a l}^{2} L^{2}}{4 \xi^{2}} .
$$

Let us calculate now the values of $S_{\mathcal{E}}$ for different possible topologies of gravitationalwave antennae.

\section{ONE-ARM SCHEME}

The simplest of possible topologies, shown in Fig. 1, consists of a single Fabry-Perot resonator excited by a pumping laser. The optimally chosen variable of the reflected beam is registered. A spectral or time-domain variational measurement [6], or any other advanced technique can be used to increase the sensitivity. Simple calculations yield that in this case

$$
S_{\mathcal{E}}(\omega)=\frac{2 \zeta^{2} \hbar \omega_{0} \overline{\mathcal{E}} \delta}{\delta^{2}+\omega^{2}}
$$

where $\omega_{0}$ is the eigenfrequency of the resonator, $\delta$ is the half-bandwidth of the resonator, $\overline{\mathcal{E}}$ is the mean value of the optical energy in the resonator, and $\zeta$ is the squeeze factor of the quantum state of the input wave $(\zeta=1$ for a coherent quantum state and $\zeta>1$ for a squeezed state). As was noted by Caves almost twenty years ago [7], the use of a squeezed state allows one to reduce the value of $\overline{\mathcal{E}}$ by the factor $\zeta^{2}$. Unfortunately,

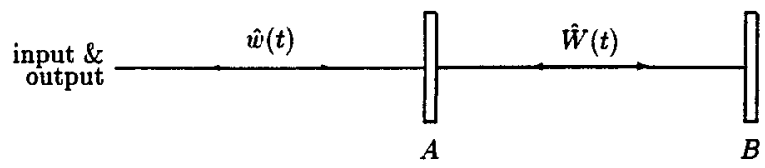

FIGURE 1. One-arm topology. 
experimental achievements in squeezing are still quite modest, so we will keep $\zeta$ in our formulas but use $\zeta=1$ (i.e. coherent state) in all numerical estimates.

Substituting formula (12) into (11) we obtain that in this case

$$
\overline{\mathcal{E}}=\frac{M \omega_{s i g n a l}^{2}\left(\delta^{2}+\omega_{s i g n a l}^{2}\right) L^{2}}{8 \xi^{2} \zeta^{2} \omega_{0} \delta},
$$

and, correspondingly,

$$
\begin{aligned}
& \bar{W}=\frac{M \omega_{s i g n a l}^{2}\left(\delta^{2}+\omega_{s i g n a l}^{2}\right) L c}{16 \xi^{2} \zeta^{2} \omega_{0} \delta} \\
& \bar{w}=\frac{M \omega_{s i g n a l}^{2}\left(\delta^{2}+\omega_{s i g n a l}^{2}\right) L^{2}}{16 \xi^{2} \zeta^{2} \omega_{0}}
\end{aligned}
$$

where $\bar{W}$ is the mean value of the optical power circulating in the resonator and $\bar{w}$ is the mean value of the input pump power.

The values of $\overline{\mathcal{E}}$ and $\bar{W}$ may be minimized by choosing for the resonator's halfbandwidth

$$
\delta=\omega_{\text {signal }}
$$

In this case

$$
\begin{aligned}
\overline{\mathcal{E}} & =\frac{M \omega_{\text {signal }}^{3} L^{2}}{4 \xi^{2} \zeta^{2} \omega_{0}} \\
& =\frac{2 \cdot 10^{8} \mathrm{erg}}{\xi^{2} \zeta^{2}}\left(\frac{M}{10^{4} \mathrm{~g}}\right)\left(\frac{\omega_{\text {signal }}}{10^{3} \mathrm{~s}^{-1}}\right)^{3}\left(\frac{L}{4 \cdot 10^{5} \mathrm{~cm}}\right)^{2}\left(\frac{\omega_{0}}{2 \cdot 10^{15} \mathrm{~s}^{-1}}\right)^{-1} \\
\bar{W} & =\frac{M \omega_{\text {signal }}^{3} L c}{8 \xi^{2} \zeta^{2} \omega_{0}} \\
& =\frac{0.75 \cdot 10^{13} \mathrm{erg} / \mathrm{s}}{\xi^{2} \zeta^{2}}\left(\frac{M}{10^{4} \mathrm{~g}}\right)\left(\frac{\omega_{\text {signal }}}{10^{3} \mathrm{~s}^{-1}}\right)^{3}\left(\frac{L}{4 \cdot 10^{5} \mathrm{~cm}}\right)\left(\frac{\omega_{0}}{2 \cdot 10^{15} \mathrm{~s}^{-1}}\right)^{-1} \\
\bar{w} & =\frac{M \omega_{\text {signal }}^{4} L^{2}}{8 \xi^{2} \zeta^{2} \omega_{0}} \\
& =\frac{1 \cdot 10^{11} \mathrm{erg} / \mathrm{s}}{\xi^{2} \zeta^{2}}\left(\frac{M}{10^{4} \mathrm{~g}}\right)\left(\frac{\omega_{\text {signal }}}{10^{3} \mathrm{~s}^{-1}}\right)^{4}\left(\frac{L}{4 \cdot 10^{5} \mathrm{~cm}}\right)^{2}\left(\frac{\omega_{0}}{2 \cdot 10^{15} \mathrm{~s}^{-1}}\right)^{-1}
\end{aligned}
$$

All known methods of overcoming the SQL for position measurements of free test masses $[6,8,9]$ apply an additional restriction on the optical energy and pumping power. This restriction arises as follows. All such methods rely on a correlation between two meter noises: the (radiation-pressure-induced) back action noise on the test masses, and the output light's shot noise. This correlation is damaged by electromagnetic fluctuations which enter the resonator wherever there is optical dissipation. Such 
fluctuations produce radiation pressure noise that cannot correlate with the shot noise, so all these schemes are limited by the level of intrinsic losses (dissipation) in the electromagnetic resonator. It can be shown that, due to this, the amount by which these methods beat the SQL cannot exceed the limit

$$
\xi=\left(\frac{\delta_{\text {intr }}}{\delta_{\text {load }}}\right)^{1 / 4}
$$

where $\delta_{\text {intr }}$ is the part of the electromagnetic resonator's half-bandwidth $\delta=\delta_{\text {intr }}+$ $\delta_{\text {load }}$ due to intrinsic losses (i.e. absorption in the mirrors and beam-splitters and nonzero transmittance of end mirrors) and $\delta_{\text {load }}$ is the part which characterizes the coupling of the resonator to the output light beam. Hence the optimization (16) is possible only if

$$
\xi>\left(\frac{\delta_{\text {intr }}}{\omega_{\text {signal }}}\right)^{1 / 4}
$$

Using the best known mirrors and the LIGO value of $L=4 \times 10^{5} \mathrm{~cm}$ one can achieve the value of $\delta_{\text {intr }} \sim 10^{-1} \mathrm{~s}^{-1}$. Hence, if $\omega_{\text {signal }} \simeq 10^{3} \mathrm{~s}^{-3}$ then condition (20) may be satisfied only if $\xi \geq 0.1$.

Any further increase of sensitivity (decrease of $\xi$ ) makes the optimization (16) impossible. Instead, the resonator's half-bandwidth will be forced to increase, with decreasing $\xi$, as

$$
\delta \simeq \delta_{\text {load }}=\frac{\delta_{\text {intr }}}{\xi^{4}}>\omega_{\text {signal }},
$$

which leads to the necessity of increasing the pumping power:

$$
\begin{aligned}
& \overline{\mathcal{E}}=\frac{M \omega_{s i g n a l}^{2} \delta_{\text {intr }} L^{2}}{8 \xi^{6} \zeta^{2} \omega_{0}} \\
& \bar{W}=\frac{M \omega_{\text {signal }}^{2} \delta_{\text {intr }} L c}{16 \xi^{6} \zeta^{2} \omega_{0} \delta} \\
& \bar{w}=\frac{M \omega_{\text {signal }}^{2} \delta_{\text {intr }}^{2} L^{2}}{16 \xi^{10} \zeta^{2} \omega_{0}}
\end{aligned}
$$

Numerical values of $\mathcal{E}, \bar{W}$ and $\bar{w}$ calculated using formulas (17)-(19) and (23)-(25) are plotted in Figs. 5, 6 and 7 correspondingly, as a functions of $\xi^{-1}$ (see the upper solid curves at the end of this paper). In these figures, LIGO parameter values are used: $M=10^{4} \mathrm{~g}, L=4 \times 10^{5} \mathrm{~cm}, \omega_{0}=2 \times 10^{15} \mathrm{~s}^{-1}$, and $\omega_{\text {signal }}=10^{3} \mathrm{~s}^{-1}$. These figures show a very strong dependence of $\mathcal{E}, \bar{W}$ and $\bar{w}$ on $\xi$ in the region to the left of the inflection point $\xi=\left(\delta_{\text {intr }} / \omega_{\text {signal }}\right)^{1 / 4}$.

This strong $\xi$ dependence $\left(\overline{\mathcal{E}} \propto \xi^{-6}, \bar{W} \propto \xi^{-6}, \bar{w} \propto \xi^{-10}\right)$ makes it practically impossible to achieve sensitivities $\xi<0.1$ using conventional interferometer topology: For $\xi=0.1$ the circulating power in the resonator arms is $\bar{W} \sim 100 \mathrm{MW}$, corresponding to a power density $\sim 300 \mathrm{~kW} / \mathrm{cm}^{2}$ in the $\sim 10 \mathrm{~cm}$-diameter light spots on each mirror's surface. This power density is already so high that it is questionable whether future mirrors will be able to handle it; and the further increase as $\bar{W} \propto \xi^{-6}$ makes it highly implausible that future mirrors will allow sensitivites better than $\xi \simeq 0.1$. 


\section{RECYCLING SCHEMES}

In the next generation of large-scale gravitational-wave antennae, recycling [10] will be used for several purposes, including reduction of the optical pump power $\bar{w}$. Meers [11] has discussed several different variants of recycling, which can be divided into two main groups, depending on the structure of the electromagnetic modes in the antenna: wide-band recyling, and narrow-band or resonant recycling. We shall illustrate these two types of recycling schemes by two concrete topologies, but the main properties in each group are independent of the concrete topology.

\section{A Wide-band recycling}

In wide-band recycling schemes (Fig. 2) the optical field in the interferometer may be regarded as a superposition of two electromagnetic modes - the input mode (also called "symmetric mode"; solid lines in Fig. 2) and the output mode (also called "antisymmetric mode"; dashed lines). The Bandwidths of these modes depend on the transmittances of the mirrors $D$ and $D^{\prime}$, correspondingly, and may be substantially different. Mirror $D^{\prime}$ may be completely absent, which corresponds to the case of simple power recycling.

All previous expressions for the mean resonator energy $\overline{\mathcal{E}}$ and circulating power $\bar{W}$ [formulas (13), (14), (17), (18), (23)] remain valid for wide-band recycling schemes, but with replacement of $\delta$ by the half-bandwidth of the output mode $\delta_{\text {out }}$. On the other hand, the value of the input (pump) power is defined by the bandwidth of the input mode:

$$
\bar{w}=2 \overline{\mathcal{E}} \delta_{i n}
$$

This allows one to decrease the input power by holding $\delta_{\text {out }} \sim \omega_{\text {signal }}$ and choosing $\delta_{i n}$ as small as possible. For example, using the best known mirrors, the value of $\delta_{i n} \sim 10^{-1} \mathrm{~s}^{-1}$ can be obtained, which allows one to decrease the input power by several orders of magnitude (see the dotted curve in Fig. 7).

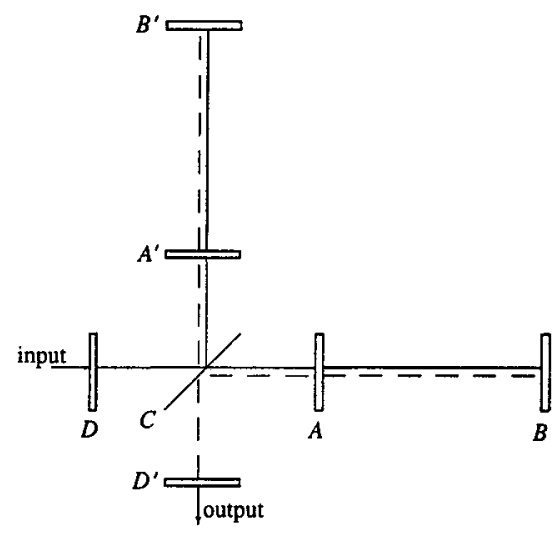

FIGURE 2. Wide-band recycling toplogy. 


\section{B Narrow-band recycling}

Narrow-band recycling schemes differ from wide-band ones only by the values of the distances between mirrors and the mirror transmittances. However, narrow-band schemes lead to a substantially different behavior of the system. Its eigenfrequencies form doublets. The frequencies in each doublet are separated from each other by a frequency difference $\Omega$ which depends on the the mirror configuration and which should be chosen close to $\omega_{\text {signal }}$. The spectral density of stored energy $S_{\mathcal{E}}$ in this case is maximimal at the frequency $\Omega$ instead of at zero frequency:

$$
S_{\mathcal{E}}=\frac{4 \hbar \omega_{0} \bar{\varepsilon} \omega^{2} \delta}{\left(\Omega^{2}-\omega^{2}\right)^{2}+\Omega^{2} \delta^{2}} .
$$

This allows one to obtain an especially high value of $S_{\mathcal{E}}$ in a narrow band in the vicinity of $\Omega$ by choosing $\Omega \gg \delta$. It can be shown that in this case

$$
\overline{\mathcal{E}}=\frac{M \omega_{s i g n a l}^{2}\left(\delta^{2}+\Delta^{2}\right) L^{2}}{8 \xi^{2} \zeta^{2} \omega_{0} \delta}
$$

where $\Delta$ is the bandwidth over which $\xi$ is close to its desired minimum value. This stored energy can be further minimized by choosing $\delta=\Delta$, so that

$$
\overline{\mathcal{E}}=\frac{M \omega_{s i g n a l}^{2} \Delta L^{2}}{4 \xi^{2} \zeta^{2} \omega_{0}} .
$$

It is evident from comparison of formulas (17) and (29) that this regime allows one to reduce the necessary value of $\mathcal{E}$, but the price is a proportional reduction of the bandwidth of the signal.

\section{INTRACAVITY SCHEMES}

The above considerations show that the Energetic Quantum Limit does not permit one to obtain sensitivities substantially better than the SQL using the traditional optical topologies of gravitational-wave antennae, even if recycling is employed.

A new method of extracting information from a gravitational-wave antenna was proposed in the article [12]. Instead of measuring the time phase shift of an output optical wave, it was suggested to detect directly the spatial phase shift of the optical field inside the antenna using some QND-type method. In this case the necessary level of uncertainty of the optical energy (2) is provided by a fluctuational redistribution of the energy between the two arms of the antenna instead of by the shot noise of the resonator energy. In principle this allows one to increase the sensitivity without increasing the mean stored energy.

Two practical realizations of this idea were considered in the articles $[13,14]$. From our point of view, the most promising of them is the "optical bar" scheme [13]. The structure of the electromagnetic modes in this case is similar to that of narrow-band recycling topologies: It has frequency doublets separated by a frequency $\Omega$, which depends on the transmittance of the central mirror. One of the possible topologies for the "optical bar" scheme is shown in Fig. 3.

In this scheme (we omit now all intermediate details) the optical fields in the two arms of the antenna work (via their radiation pressure) as mechanical springs with rigidity proportional to the optical energies $\overline{\mathcal{E}}$ stored in the two arms. The optical 
fields' rigidities move the internal mirror $C$ when a gravitational wave moves the end mirrors. This displacement may be measured relative to an additional reference mass which is not affected by the optical field in the antenna, using one of the measurement methods developed for resonant-mass gravitational-wave antennae.

The value of the displacement of mirror $C$ may be close to $L h(t) / 2$ if the stored energy $\overline{\mathcal{E}}$ is sufficiently high:

$$
\overline{\mathcal{E}} \geq \frac{m \omega_{s i g n a l}^{3} L^{2}}{2 \omega_{0}}
$$

where $m$ is the mass of the internal mirror. The structure of this expression is similar to expression (17) for traditional schemes, but it contains the mass $m$ of the internal mirror $C$ instead of the masses of the end mirrors $M$, and, which is more important, it does not depend on $\xi$. Hence, in this case it is possible to increase the sensitivity beyond the level of the SQL without any necessity to increase $\overline{\mathcal{E}}$. If, for example, $m=10^{3} \mathrm{~g}$ and the values of all other parameters are the same as above, then $\overline{\mathcal{E}} \simeq 4 \cdot 10^{7} \mathrm{erg}$.

It should be noted that internal losses in the interferometer limit the sensitivity of the intracavity schemes at the level,

$$
\xi \simeq \sqrt{\frac{\delta_{\text {intr }}}{\omega_{\text {signal }}}}
$$

[square root, by contrast with the $1 / 4$ power for conventional interferometers, Eq. (21)]; so if $\delta_{\text {intr }}=10^{-1} \mathrm{~s}^{-1}$, then the value $\xi \simeq 10^{-2}$ may be reached in principle.

\section{METHODS OF MEASUREMENT}

It is evident that for intracavity schemes the focus of the problem shifts to the local meter that measures the position of the central mirror. Intracavity schemes require high precision in this meter's measurement, exceeding the SQL. During the last twenty five years several possible methods of such measurement were proposed. We consider here briefly only one of them - the so-called " dual-resonator speedmeter" $[9,15]$. It is based on two microwave resonators, one of which couples evanescently to the position of the test mass (see Fig. 4). The sloshing of the resulting signal between the resonators,

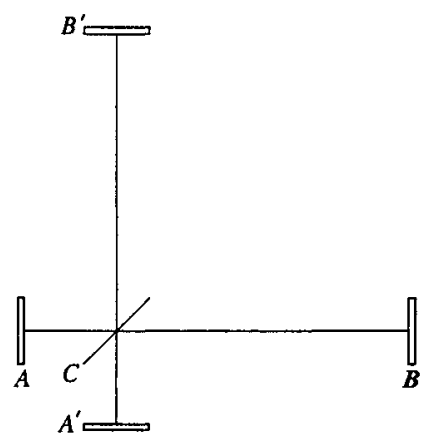

FIGURE 3. Optical bars. 
and a wise choice of where to place the resonators' output waveguide, produce a signal in the waveguide that (for sufficiently low frequencies) is proportional to the test-mass velocity, but not its position. This permits the speed meter to achieve sensitivities better than the SQL.

Unfortunately, this scheme has two major disadvantages that are typical for all similar schemes. First, when using this scheme to monitor the central mass, the level of optical energy in the antenna required to overcome the SQL has to be substantially higher than the theoretical limit (30):

$$
\overline{\mathcal{E}} \geq \frac{M \omega_{\text {signal }}^{3} L^{2}}{2 \xi^{2} \omega_{0}}
$$

and even a bit higher than the energy (17) (see the dotted curves in Figs. 5, 6, 7. This is because the speedmeter satisfies the criterion for QND measurements only when monitoring a free mass, and the internal mirror $C$ coupled to the optical field and through this field to the end mirrors is a more sophisticated object than a free mass; it has several degrees of freedom. This mirror behaves like a single solid free mass only if the optical energy is sufficiently large, which leads to the requirement (32).

The second disadvantage is an inherent property of all known devices for overcoming the SQL for position measurements of free test masses. As mentioned above, all such measurement devices are very sensitive to the level of intrinsic losses in their resonators [see formula (20) and the discussion above]. In the particular case of the speedmeter, this disadvantage limits its sensitivity to $\xi \gtrsim 1 / 3$ even if the best known microwave resonators are used [15]. An additional fee for this scheme is the necessity to use cryogenic temperatures.

The first disadvantage (abnormally high optical power) may be evaded by using a local "variational meter" [6] based on a microwave parametric transducer or on a relatively short Fabry-Perot sensor. As follows from the formula (18), the necessary circulating optical power is proportional to the length of the resonator. But for such a variational meter, the second disadvantage (a severe limitation on $\xi$ due to electromagnetic dissipation) still remains.

Two co-authors of this report think that the most promising way to evade this obstacle is to use an oscillator instead of free test mass [16]. In the case of a harmonic oscillator it is possible to overcome the SQL without using a correlation of meter noises, and thus the oscillator is free from the limitation (20).

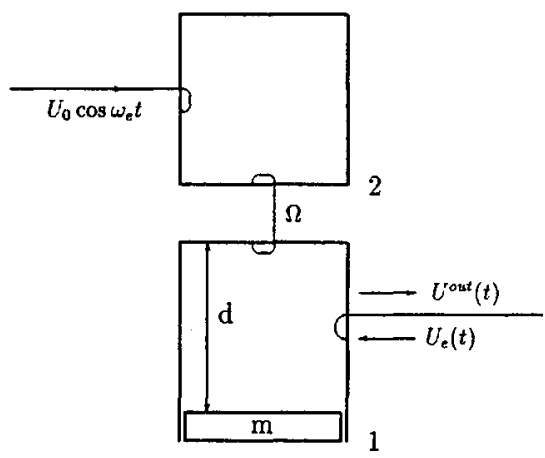

FIGURE 4. Quantum speed meter. 


\section{REFERENCES}

1. F. Ya. Khalili, Vestnik Moskovskovo Universiteta, series 3, no. 3 (1983), p. 17.

2. Yu. I. Vorontsov, F. Ya. Khalili, Vestnik Moskovskovo Universiteta, series 3, number 3 (1985).

3. V.B.Braginsky, F.Ya.Khalili, "Quantum Measurement", Chap. IX, ed.by K.S.Thorne, Cambridge University Press, 1992.

4. V. B. Braginsky, Zhur. Eksp. Teor. Fiz., 53, 1436 (1967).

5. V.B.Braginsky, F.Ya.Khalili, "Quantum Measurement", Chap. VI, ed.by K.S.Thorne, Cambridge University Press, 1992.

6. S.P.Vyatchanin, E.A.Zubova, Physics letters A201 (1995) 269.

7. C.M.Caves, Phys.Rev.D23 (1981) 1693.

8. W. G. Unruh, in Quantum Optics, Experimental Gravitation, and Measurement Theory, eds. P. Meystre and M. O. Scully, (Plenum, 1982), p. 647.

9. V.B.Braginsky, F.Ya.Khalili, Phys.Lett. A147 (1990) 251.

10. R.W.P.Drever, in "Gravitational Radiation", ads. N.Deruelle and T.Piran, NATO Adv. Inst., page 321, 1982.

11. B.J.Meers, Phys.Rev. D38 (1988) 2317.

12. V.B.Braginsky, F.Ya.Khalili, Phys. Letters A218 (1996) 167.

13. V.B.Braginsky, M.L.Gorodetsky, F.Ya.Khalili, Phys. Letters A232 (1997) 480.

14. B.Braginsky, M.L.Gorodetsky, F.Ya.Khalili, Phys. Letters A246 (1998) 485.

15. V.B.Braginsky, M.L.Gorodetsky, F.Y.Khalili, K.S.Thorne, Phys. Rev D, in press.

16. V.B.Braginsky, F.Ya.Khalili, Phys. Letters A257 (1999) 241.

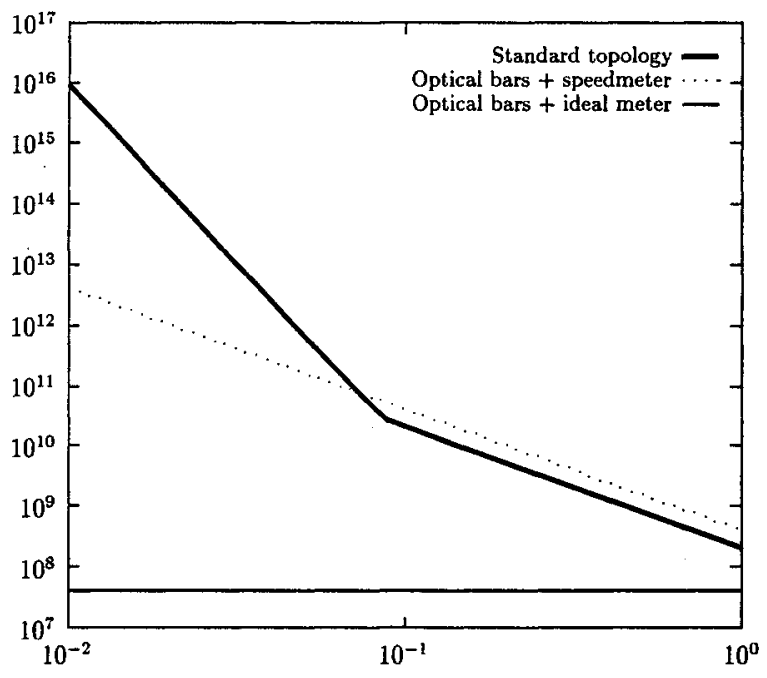

FIGURE 5. Energy as a function of $\xi$, erg. 


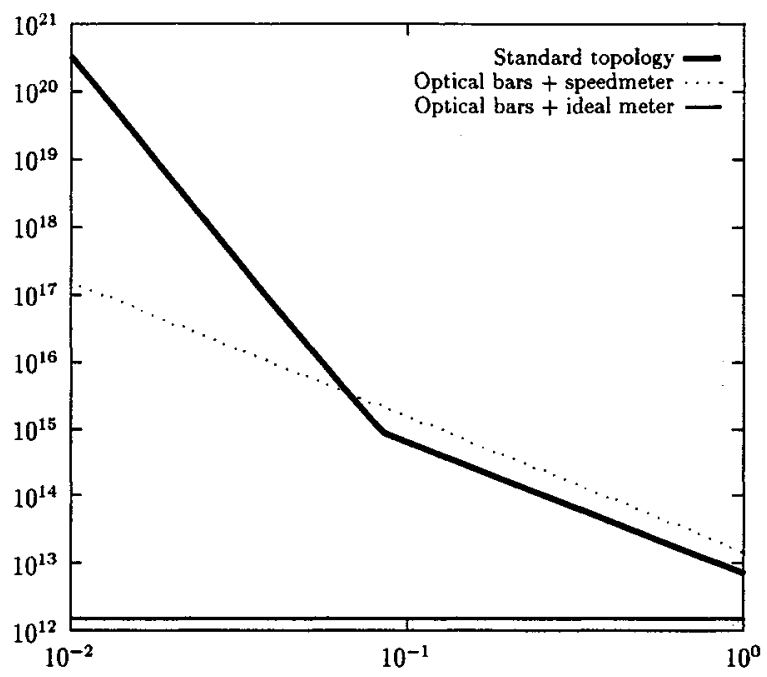

FIGURE 6. Circulating power as a function of $\xi$, erg/s.

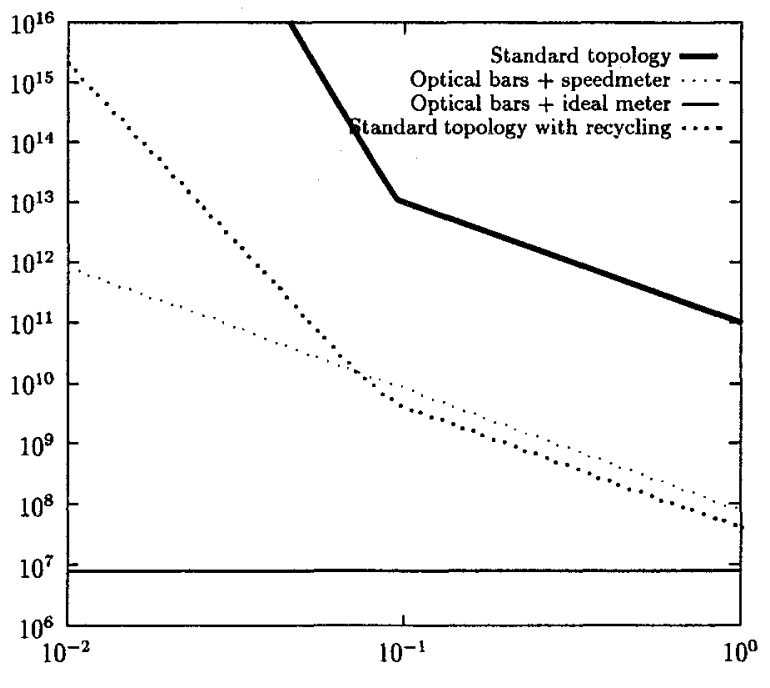

FIGURE 7. Pump power as a function of $\xi$, erg/s. 